\section{The correlation between severity of melasma with facial wrinkles in Yogyakarta, Indonesia}

\section{Dinda Saraswati Murniastuti, Kristiana Etnawati, Satiti Retno Pudjiati}

Deramatology and Venereology, Department, Faculty of Medicine, Public Health and Nursing, Universitas Gadjah Mada, Yogyakarta, Indonesia

\begin{abstract}
Melasma is a common disfiguring condition involving acquired hyperpigmentation especially on the face, for which the pathogenesis is still uncertain, however histopathological studies showed that there is not only hyperpigmentation in the epidermis, but also solar elastosis or photo-aging due to abnormality of dermal extracellular matrix which contributes to clinical wrinkles. This study aimed to examine a link between the severity of melasma and facial wrinkles as a manifestation of photoaging in a tropical area. This study was an observational study with crosssectional design, conducted in Yogyakarta, Indonesia involving 51 patients with melasma aged 30-50 years who had fulfilled the inclusion and exclusion criteria. The melasma severity was measured clinically with the modified Melasma Severity Index (mMASI),
\end{abstract} and objectively with the Melanin Index (MI) and Erythema Index (EI) assessed with Mexameter Courage Khazaka. The wrinkle severity was measured clinically by scoring from forehead horizontal lines, crow's feet, glabellar and nasolabial lines, and total scores were obtained from all of them. The correlation analysis was done statistically with Spearman's rank tests. The results showed a weak positive but not significant correlation between the mMASI score and total facial wrinkle score (r: 0.165), and a weak positive non-significant correlation between EI and total facial wrinkle score ( $\mathrm{r}: 0.06)$. There were significant positive moderate correlations between MI and total facial wrinkle score (r: 0.441 ), due to significant positive moderate correlations between MI and glabella wrinkle (r: 0.392), and between MI and nasolabial wrinkle (r: 0.339). In conclusion, a positive moderate correlation was found between MI and total facial wrinkle score, especially relating to glabellar and nasolabial wrinkles. However, there was no correlation between mMASI score and total facial wrinkle score.

\section{Introduction}

Melasma is one of the most commonly acquired hyperpigmentation especially on the face. The prevalence is high in reproductive age, with Fitzpatrick III-V skin types and people living in areas with high sun exposure. ${ }^{1}$ In Southeast Asia, melasma occurs in about $0.3 \%-4 \%$ of all patients who visit the dermatology clinics. ${ }^{2}$ In Indonesia, melasma is estimated to be around $0.25-4 \%$ of all skin disease cases. ${ }^{3}$ Melasma has a significant impact on patients because it can reduce their quality of life. ${ }^{4}$ Several studies found that a decrease of quality of life in patients with melasma based on the Dermatology Life Quality Index score (DLQI) was related to the severity of melasma. ${ }^{4,5}$

Pathogenesis of melasma is still unknown. One theory proposes that melas$\mathrm{ma}$ is a result of environmental and hormonal interactions in individuals who are genetically susceptible to melasma. ${ }^{6-8}$ The latest theory regarding the pathogenesis of melasma is that it is part of the aging process caused by ultraviolet (UV) radiation or photoaging. Location and development of melasma symptoms after sun exposure indicate the important role of ultraviolet radiation, which means that the higher exposure to sunlight, the more severe the melasma will be. ${ }^{9}$

There are various scoring systems that can be used to evaluate the severity of melasma lesions. The modified Melasma Area and Severity Index score (mMASI) has the same validity and reliability as the Melasma Area and Severity Index (MASI) score, which are some of the most popular and earliest scores used. ${ }^{10}$ Examination of spectrophotometers with Mexameter (melanin index and erythema index) and colorimeter are objective instruments that can also be used to assess the severity of pigmentation in melasma lession. ${ }^{11}$

Photoaging is a factor which explains the clinical, histological changes and functional characteristics of aging skin observed in areas that are often exposed to sunlight. Photoaging is a process that runs slowly over many years. ${ }^{12} \mathrm{UV}$ radiation induces apoptosisof the stem cells in the basal layer and hair bulbs then cause epidermal atrophy. UV exposure also makes slow wound healing and excessive melanin production was observed in old melanocytes. However, the molecular activity that triggers freckling, lentigenes and other pigmentation changes on the skin caused by photoaging is still not widely known. ${ }^{13}$
Correspondence: Kristiana Etnawati, Dermatology Department, Faculty of Medicine, Universitas Gadjah Mada, Radiopoetro Building, 3rd Floor, Sekip Utara, 55281, Yogyakarta, Indonesia

Tel.: +62 274560700

E-mail: etnawati@ugm.ac.id

Key words: melasma; photoaging; facial wrinkles; melanin index; erythema index.

Conflict of interest: The authors declare no potential conflict of interests.

Received for publication: 24 November 2019. Accepted for publication: 23 September 2020.

This work is licensed under a Creative Commons Attribution-NonCommercial 4.0 International License (CC BY-NC 4.0).

(Copyright: the Author(s), 2020

Licensee PAGEPress, Italy

Dermatology Reports 2020; 12:8390

doi:10.4081/dr.2020.8390

Many studies on photoaging have focused more on the dermis, in an effort to explain the formation of wrinkles. Until now there is no gold standard for assessing the severity of wrinkles. Some scores on the assessment of wrinkles use a photographic scale, while profilometry examination using silicon is also still widely applied as an objective examination of wrinkles. ${ }^{14} \mathrm{UV}$ radiation increases the activity of Matrix Metalloproteinase (MMPs), which degrades all extracellular matrix causing the breakdown of many collagen dermis. This effect is considered to significantly contribute to the appearance of facial wrinkles by the photoaging process. ${ }^{15-17}$

The increased activity of MMPs also plays a role in the pathogenesis of melasma, through damage of the basement membrane mediated by MMP-2 and MMP-9 due to chronic exposure of UV light, which degrades type IV and type VI collagen. Basement membrane damage facilitates the release of melanocytes and melanin into the dermis, so that melanin or melanophage can be seen in the dermis of melasma skin. ${ }^{18,19}$ Until now the photoaging theory of melasma is still controversial, but some studies found the same pathomechanisms of melasma and facial wrinkles as those involved in photoaging manifestations. This research aimed to determine the correlation between melasma and facial wrinkles in conjunction with the role of photoaging in melasma pathogenesis. 


\section{Material and Methods}

This observational research used a cross-sectional study design with melasma subjects in the Dermatology and Venereology Department, Faculty of Medicine, Public Health and Nursing, Universitas Gadjah Mada, Yogyakarta, Indonesia. Informed consent was obtained prior to the subjects' inclusion, and the study was conducted in accordance with Declaration of Helsinki. Ethical clearance and approval were obtained before the commencement of the study from the Medical and Health Research Ethics Committee of the Universitas Gadjah Mada.

The inclusion criteria of the subjects in this study were: women 30-50 years old with Fitzpatrick skin type III, IV, V who were diagnosed with melasma by a certified dermatologist based on their history and clinical examination, and subjects who were willing to participate in the study by signing informed consent forms. The exclusion criteria for subjects in this study were: pregnant and breastfeeding woman, those who suffered from thyroid disease, diabetes mellitus, reproductive disease or diseases with photosensitivity reaction, those who consume antioxidant supplements, usage of topical anti-inflammation and antioxidant medication for the last 1 month, those who use topical tretinoin or depigmentation agents for the last 3 months and topical hydroquinone for the last 6 months. Consuming hormonal contraception, and photosensitive drugs were also included as exclusion criteria.

The severity of melasma was determined clinically using the modified Melasma Severity Index (mMASI), assessed by two dermatology residents, and objectively by the Melanin Index and Erythema Index measured with Mexameter Courage Khazaka on the darkest lesion of the melasma. The severity of wrinkles was determined clinically by facial wrinkle score by applying a photographic scale on six different areas, consisting of the forehead, glabella, right and left crows feet, right and left nasolabial area. Statistical analysis was done using Spearman's rank correlation test with $p$ value $<0.05$ considered significant.

\section{Results}

\section{Characteristics of the subjects}

This research was conducted on 51 subjects diagnosed with melasma in the Yogyakarta area. All of the participants were woman with age between $30-50$ years old and mean age of $45.5 \pm 4.35$. The types of melasma consisted of centrofacial (61\%) and malar $(39 \%)$, whereas there was no mandibular type.

Correlation between severity melasma and facial wrinkles

The correlation between the severity of melasma and the wrinkle facial score in each location of the face was described in Table 1. Results showed that there was a moderate positive and statistically significant correlation between MI and the wrinkle score in glabellar wrinkle also between MI and wrinkle score in nasolabial wrinkle. This finding consequently led to a moderate positive and statistically significant correlation between MI and the total facial wrinkle score. However, there was also a positive correlation found but it was not statistically significant between mMASI and total facial wrinkle score.

\section{Discussion}

Previous studies found higher solar elastosis material as a sign of the photoaging process in melasma lesions compared to normal perilesional skin through biopsy examination, ${ }^{20,21}$ however no studies discussed the correlation between the several signs of photoaging in melasma and clinically measured areas of facial wrinkles.

Solar elastosis results from long-term sun exposure observed histopathologically, and it has various features of clinical manifestations. These clinical and histological changes are often referred to as dermatoheliosis. Photoaging is said to be a synonym for dermatoheliosis, but many authors limit its use to initial changes that can be seen, and wrinkles are one manifestation among other clinical forms of photoaging. From this explanation it can be concluded that the correlation of solar elastosis in patients with melasma, cannot be directly related to wrin- kles as a clinical manifestation. ${ }^{22,23}$ This can also be applied to the correlation result between melasma severity by mMASI score and erythema index with facial wrinkle score, for which no significant correlation result was found, however another objective measurement of melanin index as a sign of objective measurement for melasma severity showed positive moderate and statistically significant correlation with facial wrinkle score. ${ }^{22}$

The study by Tsukahara et al. ${ }^{22}$ described the correlation between solar elastosis with the formation of wrinkles on the forehead and on the lateral canthus (crows feet). The study concluded that solar elastosis was identifiable and has a role in early development of wrinkles on the forehead and crows feet, but in deep wrinkle, evidence of solar elastosis was not found. Other factors that may affect this condition are pressure due to facial muscles movement and the formalin's cadaver effect.

In this study, a Mexameter was used as a tool to measure the melanin and erythema index as the specific objective parameters in understanding the level of melanin and the vascular involvement in the melasma lesion. ${ }^{24}$ Moderate positive and statistically significant correlation was found in the analysis of the melanin index with facial wrinkles score based on the photographic scale. The facial wrinkle score by photographic scale includes wrinkle scoring on four areas of the face, so it does not reflect the wrinkles caused by photoaging only.

In more detailed correlation analysis between the melanin index and facial wrinkle scores in each area, a moderate positive and statistically significant correlation was found in the glabella and nasolabial folds. According to Lamperle et al. ${ }^{8}$, wrinkles in the glabellar area and nasolabial folds were included as mimetic wrinkles, which were a combination of repetitive movements of facial muscle expressions and due to sun

Table 1. Correlation between facial wrinkle score and severity of melasma.

\begin{tabular}{lccc} 
Type of wrinkle & mMAST & Melanin Index (MI) & Erythema Index (EI) \\
Forehead & $\mathrm{r}:-0.068$ & $\mathrm{r}: 0.180$ & $\mathrm{r}: 0.137$ \\
& $p: 0.635$ & $p: 0.206$ & $p: 0.338$ \\
Glabella & $\mathrm{r}: 0.194$ & $\mathrm{r}: 0.392$ & $\mathrm{r}: 0.036$ \\
& $p: 0.173$ & $p: 0.004^{*}$ & $p: 0.803$ \\
\hline Lateral Eye (crow's feet) & $\mathrm{r}: 0.023$ & $\mathrm{r}: 0.195$ & $\mathrm{r}: 0.092$ \\
& $p: 0.871$ & $p: 0.171$ & $p: 0.522$ \\
Nasolabial Folds & $\mathrm{r}: 0.063$ & $\mathrm{r}: 0.339$ & $\mathrm{r}:-0.011$ \\
& $p: 0.660$ & $p: 0.015^{*}$ & $p: 0.940$ \\
\hline Total facial wrinkle score & $\mathrm{r}: 0.165$ & $\mathrm{r}: 0.441$ & $\mathrm{r}: 0.106$ \\
& $p: 0.246$ & $p: 0.001^{*}$ & $p: 0.459$
\end{tabular}

r: correlation coefficient, $p$ : significant value, with $p<0.05$ considered to be signficant $(*)$. 
exposure based on dermal elastosis that was found in the area.

The wrinkles with the surrounding skin comprise the Wrinkle Unit that plays a role in the function of a clinically defined system called, the 'Functional Psycho-NeuroMuscular System of the Face for Protection, Expression and Communication'. The functions of the Wrinkle Unit are expressing emotions, protection of orifices, especially of the eyes from the sun, trauma (by fast movement of eyebrows and eyelids), and also as a reservoir of skin tissue. ${ }^{25}$ This system could explain the moderate positive correlation result between the melanin index and wrinkles in the glabellar area and nasolabial folds, showing that high sun exposure in that area is reflected by high melanin index, which may be caused by repeated spontaneous movements of facial muscles related to the Wrinkle Unit, especially the glabellar area.

There are several weaknesses in this study. One of the confounding factors that were not controlled in the study involves total amount of sun exposures in each subject. Stress or anxiety status is also considered to be one of the factors involved in expressions related facial muscle movement. Research about stress or psychological status related to melasma and facial wrinkle needs to be done to support the results of this study.

\section{Conclusions}

There was no significant correlation found between melasma severity measured by mMASI and erythemal index with the facial wrinkle score. Moderate positive correlations were found between melanin index and total facial wrinkles score. Analysis for each area of the face revealed that wrinkles at glabellar and nasolabial folds, which are considered to be mimetic wrinkles, have positive correlation with MI, in which higher MI in melasma lesions strongly correlates with higher wrinkle scores in both areas. These results reflect conclusive findings that sun exposure has a significant role in increasing skin melanin and also facial skin wrinkles.

Considering Indonesia as a country that has various ethnic groups with different skin types, and also experiences high sun exposure, further research is needed to study the role of skin type and ethnicity in the type and development of facial wrinkles of Indonesian people.

\section{References}

1. Sheth VM, Pandya AG. Melasma: a comprehensive update: part 1. J Am Acad Dermatol 2010;65:689-97.

2. Rathi SK, D'Souza P. Epidemiology Melasma. In: Lahiri K, Charttejee CM, Sarkar R. (Eds). Pigmentory Disorders: A Comprehensive Compedium. New Delhi: Jaypee Brother Medical 2014;281-2.

3. Soepardiman L, Ruswan SA. Epidemiology of melasma in Asian Countries. In: Pigmentary Disorders from Global Perspective. Abstract book and final program of the satellite meeting of the 19th World Congress of Dermatology, Bali; 1997.

4. Handel AC, Miot LDB, Miot HA. Melasma: a clinical and epidemiological review. Ann Bras Dermatol 2014;89:771-82.

5. Ikino JK, Nunes DH, da Silva VPM, Frode TS, Sens MM. Melasma and assessment of the Quality of Life in Brazilian Women. Ann Bras Dermatol 2015;90:196-200.

6. Nicolaidou E, Katsambas AD. Pigmentation disorders: hyperpigmentation and hypopigmentation. Clin Dermatol 2014;32:66-72.

7. Pandya AG, Hynan LS, Bhore R, Riley FC, Guevera IL, Grimes P, et al. Reliability assessment and validation of the Melasma Area and Severity Index (MASI) and a new modified MASI scoring method. J Am Acd Dermatol 2011;64:78-83.

8. Lemperle G, Holmes RE, Cohen SR, Lemperle SM. A classification of facial wrinkles. Plastic Reconstructive Surg 2001;108:1735.

9. Young Lee Ai. An updated review of melasma pathogenesis. Dermatologica Sinica 2014;32:233-9.

10. Abou-Taleb DA, Ibrahim AK, Youssef EMK, Maubasher A. Reliability, validity, and sensitivity to change overtime of the Modified Melasma Area and Severity Index Score. Dermatol Surg 2017;43:210-7.

11. Clarys P, Alewaeters K, Lambrecht R, Barel AO. Skin color measurements: comparison between three instruments: The Chromameter (R), the DermaSpectrometer (R) and the Mexameter (R). Skin Res Technol 2000;6,230-8.

12. Jeong JH, Fan Y, You GY, Choi TH, Kim S. Improvement of photoaged skin wrinkles with cultured human fibroblasts and adipose-derived stem cells: a comparative study. J Plastic
Reconstructive Aesthetic Surg 2015;68:372-81.

13. Gilchrest BA. Photoaging. J Investig Dermatol 2013;133:E2-E6.

14. Fabi SG, Massaki A, Eimpunth S, Pogoda J, Goldman MP. Evaluation of microfocused ultrasound with visualization for lifting, tightening, and wrinkle reduction of the décolletage. J Am Acad Dermatol 2013;69:965-71.

15. Quan T, Qin Z, Xia W, Shao Y, John J, Voorhees, Gary J. Matrix-degrading metalloproteinases in photoaging. J Investig Dermatol Symp Proc 2009;14:20-4.

16. Kim HH, Lee MJ, Lee SR, Kim KH, Cho KH, Eun HC, Chung JH. Augmentation of UV-induced skin wrinkling by infrared irradiation in hairless mice. Mech Ageing Dev 2005;126: 1170-7.

17. Imokawa G. Mechanism of UVBinduced wrinkling of the skin: paracrine cytokine linkage between keratinocytes and fibroblasts leading to the stimulation of elastase. J Investig Dermatol Symp Proc 2009;14:36-43.

18. Kwon SH, Hwang YJ, Lee KS, Park KC. Heterogeneous pathology of melasma and its clinical implications. Int J Mol Sci 2016;17:824.

19. Amano S. Characterization and mechanisms of photoageing-related changesin skin. Damages of basement membrane and dermal structures. Experiment Dermatol 2016;25:14-9.

20. Kang WH, Yoon KH, Lee ES, Kim J, Lee KB, Yim H, Sohn S. Melasma: histopathological characteristics in 56 Korean patients. Br J Dermatol 2002; 146:228-37.

21. Hernandez-Barrera R, Torres-Alvarez B, Castanedo-Cazares JP, Oros-Ovalle C, Moncada B. Solar elastosis and presence of mast cells as key features in the pathogenesis of melasma. Clin Experiment Dermatol. 2008;33:305-8.

22. Tsukahara K, Tamatsu Y, Sugawara Y, Shimada K. Morphological study of the relationship between solar elastosis and the development of wrinkles on the forehead and lateral canthus. Arch Dermatol 2012;148:913-7.

23. Calderone DC, Fenske NA. The clinical spectrum of actinic elastosis. J Am Acad Dermatol 1995;32:1016-24.

24. Pandya A, Berneburg M, Ortonne J, et al. Guidelines for clinical trials in melasma. Br J Dermatol 2007;156:218.

25. Hatzis J. The wrinkle and its measurement - A skin surface profilometric method. Micron 2004;35:201-19. 